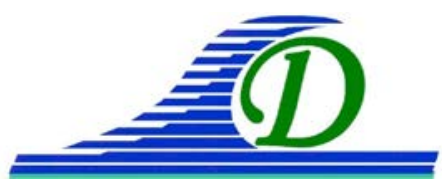

XIII ${ }^{\text {èmes }}$ Journées Nationales Génie Côtier - Génie Civil Dunkerque, 2-4 juillet 2014

DOI:10.5150/jngcgc.2014.085 C Editions Paralia CFL

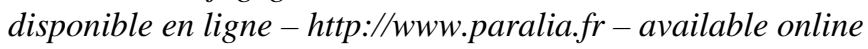

\title{
Utilisation de la courantographie radar pour la quantification des ressources hydrocinétiques et l'analyse d'asymétrie des courants sur un site d'énergies renouvelables en mer d'Iroise
}

\author{
Alexei SENTCHEV ${ }^{1}$, Maxime THIEBAUT ${ }^{1}$
}

\author{
1. Université du Littoral - Côte d'Opale, Laboratoire d'Océanologie et Géosciences \\ (UMR8187), 62930 Wimereux, France. \\ alexei.sentchev@univ-littoral.fr ; maxime.thiebaut@etudiant.univ-lille1.fr
}

\section{Résumé :}

Les mesures de courants par radar en mer d'Iroise sont utilisées pour mettre en évidence la complexité de la dynamique de marée autours des îles Ouessant - Molène qui se traduit par l'intensification des courants, le changement de la polarisation d'ellipses de marée et de l'asymétrie de vitesses. Nous avons démontré une structuration dans la distribution des ellipses de courant, identifié des régions dominées par le courant de flot et de jusant, quantifié l'asymétrie des vitesses et la puissance de l'écoulement.

Mots-clés : Courant de marée, mesures radar, énergie renouvelable, mer d'Iroise

\section{Introduction}

La sélection de sites pour des projets EMR nécessite une bonne connaissance de la dynamique régionale côtière et l'application d'une méthodologie pertinente de quantification des ressources hydrocinétiques. Parmi les critères de sélection on trouve d'abord l'amplitude de courant et l'occurrence de vitesses au-dessus d'un certain seuil. Peu d'attention est généralement prêtée au degré d'asymétrie des courants. L'asymétrie se traduit non seulement par une différence des vitesses entre le flot et le jusant et la différence du niveau de la mer, mais affecte également d'autres variables physiques, telle que la turbulence et le cisaillement (KOROTENKO et al., 2012), qui ont une importance capitale pour le bon fonctionnement de récupérateurs. La distorsion des courbes de niveau et de vitesse de courant est liée à la présence des composantes nonlinéaires générées par diverses interactions, lorsque l'onde de marée se propage en région peu profonde avec de gradients de bathymétrie importants et le trait de côte découpé (e.g., LE PROVOST, 1991). Les modèles de circulation côtière sont capables de reconstituer l'évolution du niveau de la mer avec une bonne précision (RAMOS et al., 2013). Cependant la modélisation des courants demeure une tache plus ardue.

La courantographie radar, devenue routinière dans de nombreuses régions côtières (e.g., PADUAN \& WASHBURN, 2013), s'avère particulièrement attrayante pour la caractérisation des ressources hydrocinétiques sur des sites dédiés aux énergies marines renouvelables. Elle offre une vision précise de l'écoulement, de son hétérogénéité spatiale et temporelle. Les mesures radars constituent un moyen d'analyse puissant et 


\section{Thème 5 - Énergies et ressources marines}

nous présenterons dans cet article une nouvelle approche méthodologique visant à établir une description détaillée de l'évolution des courants dans un secteur à fort potentiel énergétique en mer d'Iroise (nord Gascogne).

\section{Méthodologie de l'étude}

\subsection{Mesures radar des courants superficiels}

Deux radars océanographiques HF "Wellen Radar" (WERA) sont installés depuis juillet 2005 sur la côte de la Bretagne occidentale (figure 1). Les mesures de courants sont destinées principalement à la validation des modèles numériques de circulation et à l'estimation des propriétés de l'écoulement (ARDHUIN et al., 2009, SENTCHEV et al., 2013). Le principe de la mesure repose sur l'effet Doppler. L'interaction résonnante entre les ondes électromagnétiques émises par le radar et les vagues de surface, dont la vitesse est modifiée par le courant marin, crée un décalage Doppler $\Delta f$. La conversion de décalage en vitesse de courant $V$ est faite directement au travers la relation $V=2 \Delta \mathrm{f} / \lambda$ (PRANDLE, 1991), $\lambda$ étant la longueur d'onde utilisée par le radar. En utilisant ce principe, le radar mesure la vitesse de courant superficiel en projection sur un certain nombre de portes en distance et de directions azimutales avec une précision supérieure à $5 \mathrm{~cm} / \mathrm{s}$. Pour la configuration en mer d'Iroise, les mesures représentent les vitesses de surface (jusqu'à $0.7 \mathrm{~m}$ de profondeur) moyennées dans le temps sur $20 \mathrm{~min}$ et reparties dans l'espace sur une grille distance-azimut d'une résolution de $1.5 \mathrm{~km}$ et de $2^{\circ}$ respectivement.

Les vitesses radiales, mesurées par deux radars distants d'une cinquantaine de km (figure 1), sont ensuite interpolées aux points de grille régulière et combinées de manière à produire des cartes vectorielles des courants dans une zone de recouvrement des radars (SENTCHEV et al., 2013). Nous avions à notre disposition des séries annuelles de vitesses (année 2007). Cependant, l'analyse s'est portée sur une période plus courte, à savoir, avril-mai 2007, caractérisée par une très grande variation du coefficient de marée. Pour cette même période, nous avons appliqué une correction de vitesses mesurées par radar de l'effet de vagues, selon la méthode proposée par (ARDHUIN et al., 2009). Les comparaisons des mesures radar avec des trajectoires de bouées dérivantes, des mesures ADCP et des sorties modèles ont été effectuées par de différents auteurs (ARDHUIN et al., 2009, LE BOYER et al., 2009; MULLER et al., 2009) et ont montré une forte cohérence entre elles.

\subsection{Méthodes d'estimation et d'analyse}

Pour caractériser la circulation en mer Iroise, dominée essentiellement par la marée, nous avons appliqué la méthode d'analyse des composantes principales PCA (EMERY \& THOMSON, 2001) aux séries temporelles de vitesse de courant. L'estimation des valeurs propres de la matrice de covariance des composantes $u$ et $v$ de vitesse en un 


\section{XIII ${ }^{\text {èmes }}$ Journées Nationales Génie Côtier - Génie Civil \\ Dunkerque, 2-4 juillet 2014}

point quelconque de l'espace permet d'estimer les paramètres synthétiques de l'ellipse de courant et de quantifier ainsi la dynamique de la marée : la force de courant (axe majeur), la direction de courant dominant (orientation de l'axe) et l'anisotropie de courant (ellipticité). A titre d'exemple, nous présentons sur la figure 2 les ellipses synthétiques de courant de marée de vive-eau (VE) et de morte-eau (ME) au point géographique situé au nord-ouest de Ouessant (cf. figure 1 pour la position du point). Les séries temporelles de composantes $u$ et $v$ sont aussi représentées sur la figure 2. Les valeurs de vitesse de courant de flot et de jusant pour les deux périodes de marée (VE et ME) sont indiquées par les points de couleur rouge et bleu. Les demi-axes majeur et mineur sont déduits de la variance de vitesse par rapport au barycentre de l'ellipse. En utilisant cette technique il est possible d'estimer les vitesses moyennes et maximales de jusant ou de flot en tout point de grille et pour toute autre période. Nous avons procédé ensuite à l'estimation du coefficient d'asymétrie $a=\langle V\rangle_{\text {flot }}\left\langle\langle V\rangle_{\text {jusant }}\right.$ en utilisant les valeurs moyennes de vitesse $\langle V\rangle$ de flot ou de jusant (cercle plein et vide sur la figure 2) pour deux périodes d'analyse - VE et ME.

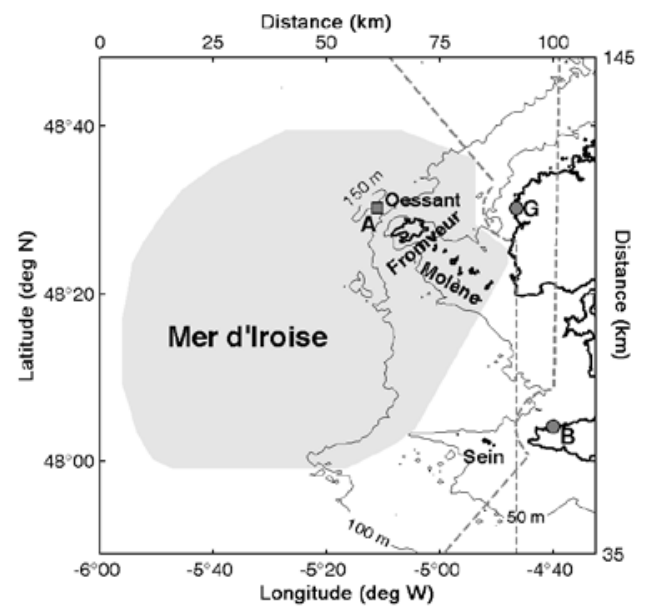

Figure 1. Mer d'Iroise avec la bathymétrie (lignes grises), la couverture radar (trait rompu), la grille d'interpolation en gris, et la position des radars (cercles gris). Le rectangle indique la position d'un point (A) utilisé pour illustrer la méthode d'estimation de l'asymétrie. Les points $G$ et $B$ représentent les sites radar (Garchine et Brezellec).

Il convient de préciser que la méthode PCA ne donne pas d'information sur le sens de rotation du vecteur courant. Pour compléter l'étude, nous avons appliqué l'analyse rotationnelle aux séries temporelles des vitesses (GONELLA, 1972). Les valeurs du pic dominant le spectre à la fréquence semi-diurne, $S_{-}$(composante indirecte) et $S_{+}$ (composante directe) sont utilisées pour estimer le coefficient rotatoire $r$ selon la relation : $r=\left(S_{+}-S_{-}\right) /\left(S_{+}+S_{-}\right) . r$ varie de -1 à +1 pour une rotation indirecte/directe. 


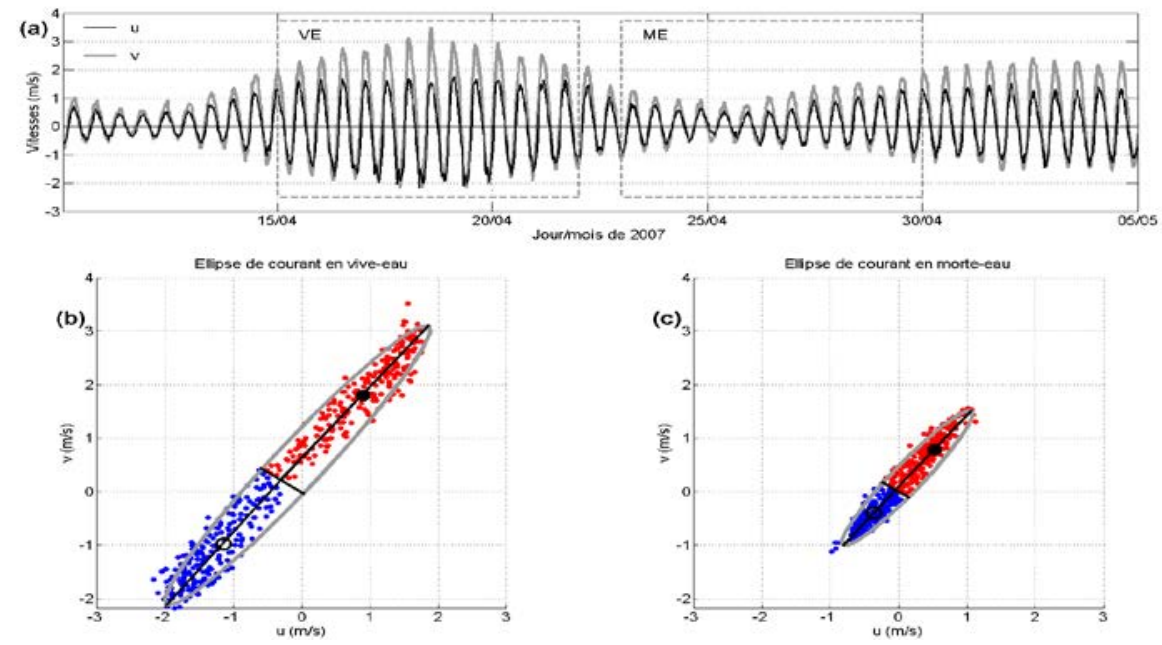

Figure 2. Séries des composantes u et $v$ de vitesse mesurée par radar et leur représentation sous forme d'ellipse de courant pour la période de vive-eau (VE) et de morte-eau (ME). Points rouges et bleus sont les vitesses de flot et de jusant avec leurs moyennes (cercle plein et vide).

\section{Résultats}

La figure 3a montre l'amplitude maximale et moyenne (sur 7 jours en VE) des courants dans le secteur d'Ouessant - Molène. Les ellipses (figure 3b) indiquent la direction des courants de marée. Le sens de rotation des vecteurs courant, ou polarisation, est précisé par le code de couleur : rouge, pour la rotation directe et bleu, pour la rotation indirecte. L'onde de marée se propage depuis le Golfe de Gascogne en direction N-NE, contourne les îles et se dirige vers La Manche. Les gradients de bathymétrie et la présence des îles induisent l'accélération locale ce qui explique l'augmentation de la vitesse de courant aux alentour de Ouessant et dans le Fromveur. Le sens de rotation des vecteurs courant change, passant de l'indirect au large au direct au voisinage des îles. Ce changement ne résulte pas de l'effet de frottement sur le fond, car la profondeur dépasse largement 50 $\mathrm{m}$, mais peut être expliqué par l'interaction de l'onde de marée avec l'île et la génération de la vorticité positive au sud de l'île pendant le jusant. D'après SENTCHEV et al. (2013), cette vorticité positive, advectée vers l'ouest après la renverse du courant, peut contraindre le sens de rotation du vecteur vitesse au sud d'Ouessant. Le phénomène inverse se produit au nord de l'île pendant le flot où l'on retrouve la rotation indirecte des courants de marée. Les vitesses de courant maximum avoisinent $4 \mathrm{~m} / \mathrm{s}$ aux deux endroits : dans le Fromveur et au NO d'Ouessant. Dans cette zone, en période de viveeau, la vitesse moyenne reste supérieure à $1.5 \mathrm{~m} / \mathrm{s}$ ce qui rend cet endroit particulièrement attrayant pour l'installation des hydroliennes. Les propriétés de l'écoulement dans le passage du Fromveur sont globalement similaires : avec la vitesse maximale de courant atteignant $4 \mathrm{~m} / \mathrm{s}$ et la vitesse moyenne supérieure à $1 \mathrm{~m} / \mathrm{s}$ (figure 3a). 


\section{XIII ${ }^{\text {èmes }}$ Journées Nationales Génie Côtier - Génie Civil \\ Dunkerque, 2-4 juillet 2014}

La distribution cumulative des occurrences de vitesse est une autre grandeur généralement requise pour la recherche de meilleurs emplacements de récupérateurs. La figure 4 montre cette distribution en 4 points particuliers situés aux endroits où l'amplitude de courant est élevée : au nord d'Ouessant et dans le Fromveur (cf. Fig. 3a pour la position des points). Deux d'entre eux (A et C) montrent une distribution élevée de grandes valeurs de vitesse pendant un cycle semi-mensuel. La vitesse de courant supérieure à $1 \mathrm{~m} / \mathrm{s}$ est observée à plus de $50 \%$ du temps au nord d'Ouessant (pt. A) et $65 \%$ du temps dans le Fromveur (pt. C). À mesure que l'on s'éloigne du centre du Fromveur, l'amplitude de vitesse de courant et l'occurrence des vitesses élevées décroît sensiblement (Fig. 4). En utilisant les mesures radar, il est donc possible d'établir des cartes de distribution des occurrences (non présentées) pour de périodes différentes.

Les distributions d'amplitudes et des occurrences de vitesse ont montré une certaine ressemblance des deux sites : nord d'Ouessant et Fromveur. Cependant, nous avons pu constater une différence notable entre eux. Elle se traduit par une asymétrie des courants de jusant et de flot profondément marquée. Comme l'on peut constater sur la figure 5, cette asymétrie persiste au cours du cycle de marée semi-mensuel. Au nord du Fromveur les valeurs du coefficient d'asymétrie restent, en moyenne, supérieures à 2. Ici, la vitesse de courant de flot est largement plus grande que celle de jusant. Cette différence diminue à mesure que l'on se déplace vers le centre du Fromveur. La courbe de vitesse s'équilibre. Mais une autre distorsion de la courbe de vitesse apparaît à la sortie du Fromveur. Dans une zone relativement étendue, couvrant le sud de l'île d'Ouessant, les valeurs du coefficient d'asymétrie diminuent jusqu'à 0.5 et le régime de l'écoulement est dominé par le jusant. Plus vers le large, dans une bande à largeur variable, orientée nord-sud à l'ouest des îles, les courants sont dominés par la marée de flot ( $a>1$ avec un maximum 1.6).
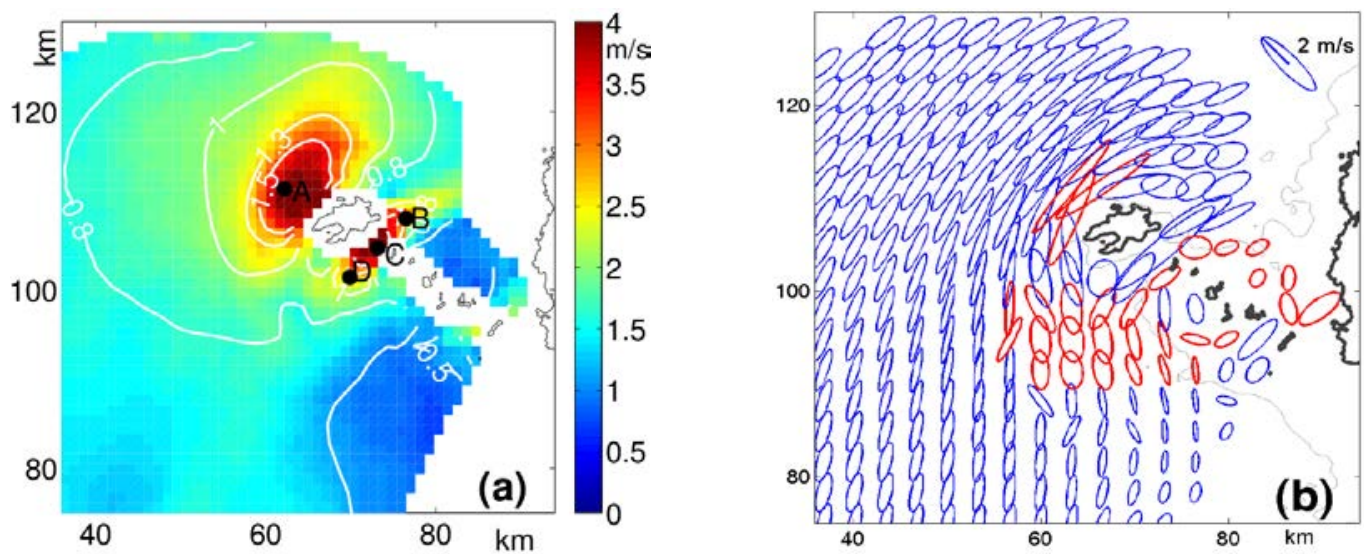

Figure 3. Amplitude moyenne (contours blancs) et maximale (code couleur) des courants de surface en vive-eau (a). Ellipses de courant de marée en vive-eau (b). Le sens de rotation du vecteur vitesse (directe ou indirecte) est représenté par la couleur

rouge et bleue. A-D sont les points de grille utilisés dans l'analyse statistique. 


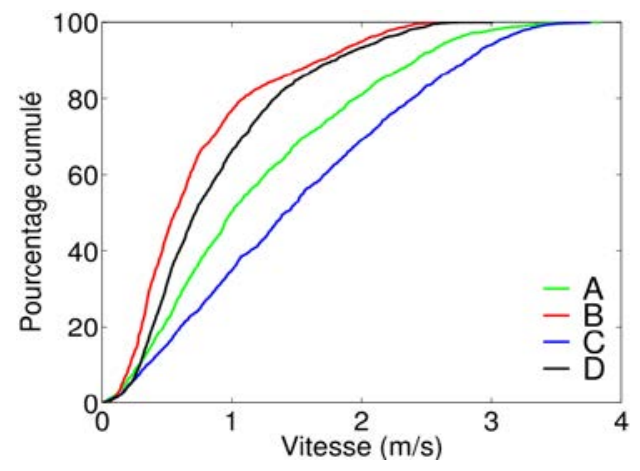

Figure 4. Distribution cumulée des occurrences de vitesse de courant en 4 points particuliers (A-D) pendant un cycle de marée semi-mensuel au mois d'avril 2007.
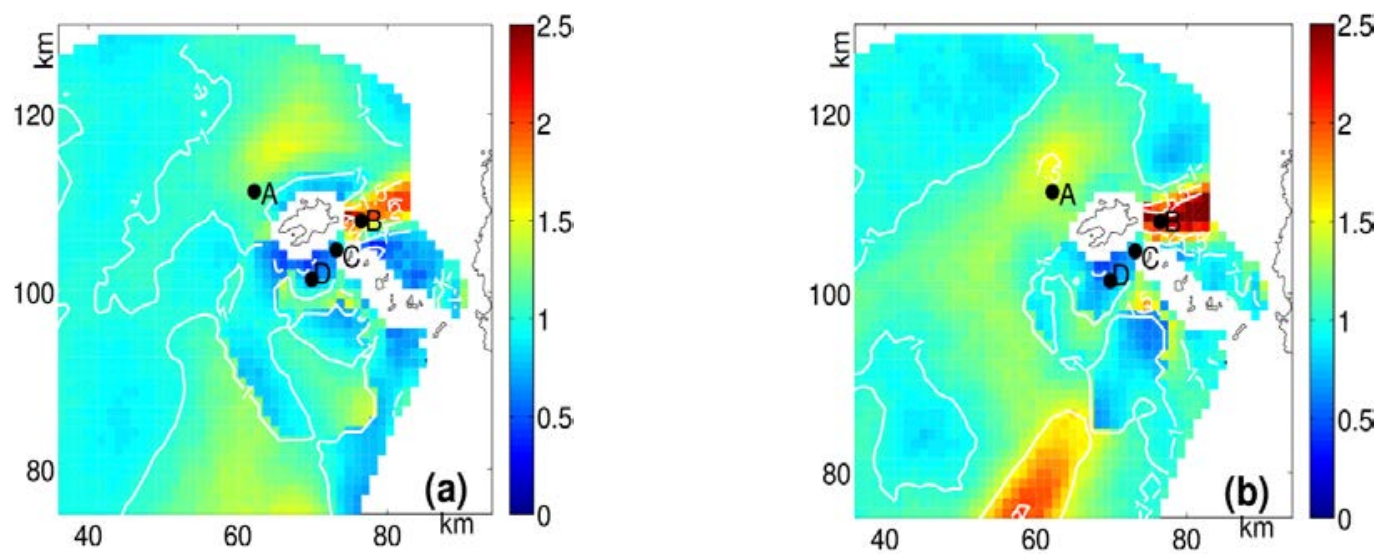

Figure 5. Valeurs du coefficient d'asymétrie a en vive-eau (a) et en morte-eau (b).

L'asymétrie des vitesses de courant pourrait avoir un impact crucial sur l'énergie récupérable si les machines ont des modes de fonctionnement différents en flot et en jusant. Nous présentons sur la figure 6 les séries temporelles de vitesse et d'énergie disponible $(P)$ en trois points situés sur l'axe du Fromveur (voir figure 3 pour la position des points). Nous utilisons une simple formulation d'Actuator Disk (e.g., HOULSBY et al., 2008), $P=0.5 \rho C_{P} A u^{3}$, avec $\rho$ la masse volumique de l'eau de mer, $u$ la vitesse de courant minorée de $20 \%$ pour tenir compte de sa décroissance verticale (e.g., PRANDLE, 1991), $C_{P}=0.59$ et enfin $A=78 \mathrm{~m}^{2}$ qui est la surface du disque de $10 \mathrm{~m}$ de diamètre. La valeur symbolique, $1 \mathrm{~m} / \mathrm{s}$, indiquée par un trait horizontal, correspond à la vitesse de mise en marche de certains systèmes. La diminution de vitesse en surface de $20 \%$ permet d'approcher la vitesse de courant moyen (moyenne verticale) et rend ainsi l'estimation de puissance à la hauteur des machines plus réaliste. Les courbes montrent que le niveau de puissance dépasse un seuil symbolique $(0.025 \mathrm{MW})$ pendant plusieurs heures par jour durant le cycle de marée VE-ME. Une autre caractéristique remarquable de cette distribution est le déphasage proche de $\pi$ entre le point $B$ et le point $\mathrm{D}$. Ce phénomène, qui peut paraître gênant pour la conception d'un réseau de récupérateurs, semble pouvoir offrir un avantage en cas d'utilisation de systèmes à axe horizontal 


\section{XIII ${ }^{\text {èmes }}$ Journées Nationales Génie Côtier - Génie Civil \\ Dunkerque, 2-4 juillet 2014}

monodirectionnel (comme Triton de TidalStream ou Sabella D03). La superposition des deux courbes de puissance (figure 6d) montre qu'il est possible non seulement d'équilibrer la production d'énergie durant la journée mais aussi d'étendre la puissance récupérable jusqu'à $45 \%$ de temps.

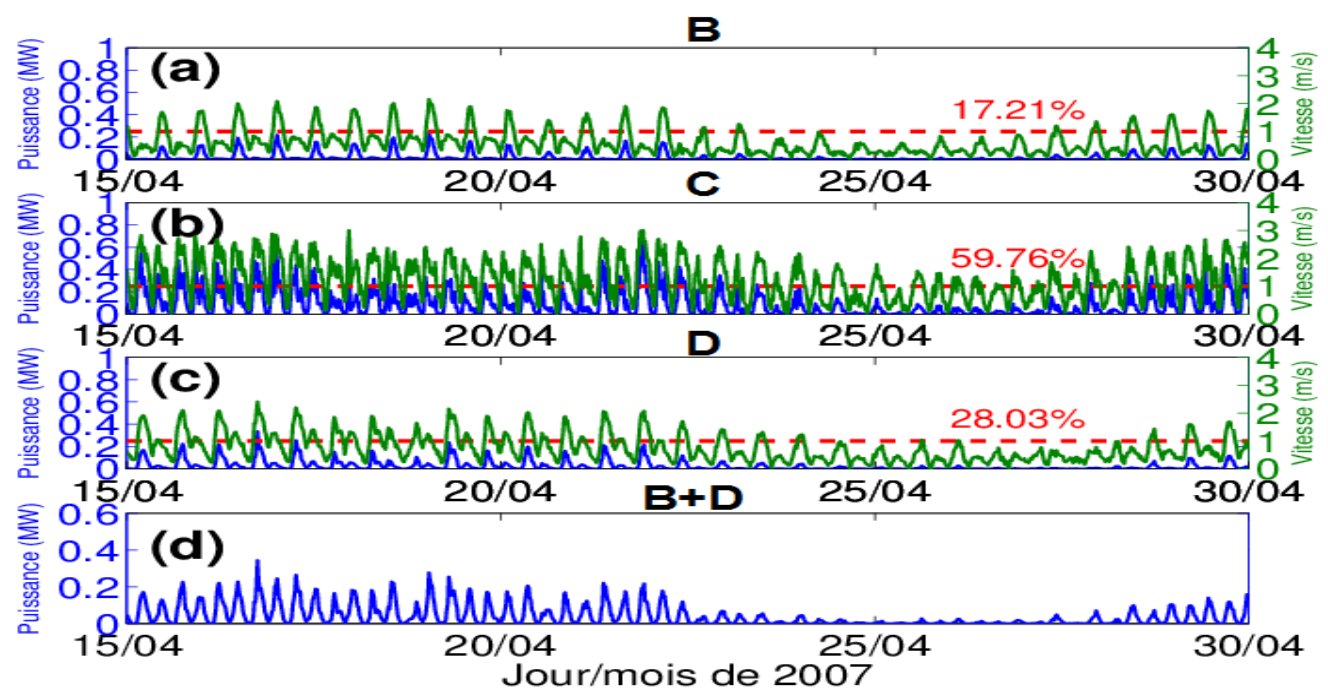

Figure 6. Séries temporelles de la force de courant $|V|$ (en vert) et de la puissance $P$ disponible dans l'écoulement (en bleu) aux points $B, C$, et $D$ dans le Fromveur.

\section{Conclusions}

L'analyse des mesures de courant par radar HF en mer d'Iroise a permis de mettre en évidence la complexité de la dynamique de marée autours des îles Ouessant - Molène qui se traduit, en particulier, par l'intensification des courants, le changement de polarisation et d'asymétrie. Différents mécanismes sont à l'origine de ces phénomènes.

Nous voulions focaliser sur l'étude d'asymétrie et de variabilité temporelle des courants. L'interaction entre les ondes principales (M2, S2, ...) et leurs harmoniques d'ordre supérieures (M4, MS4, M6, ...) est à l'origine d'une asymétrie de courant de marée (LE PROVOST, 1991). Nous avons quantifié l'asymétrie par le biais de l'estimation d'un rapport de vitesse de jusant et de flot (coefficient $a$ ), démontré la structuration assez complexe de la zone et l'existence des structures spatiales cohérentes dans la distribution de $a$. Le passage de Fromveur représente un cas très particulier. Les valeurs du coefficient varient de 0.5 au sud jusqu'à 2.5 à la sortie nord du passage avec la courbe de vitesse assez équilibrée en son centre. SENTCHEV et al., (2013) ont démontré la présence dans le Fromveur des harmoniques non-linéaires mixtes sixdiurnes (2MN6, 2MS6) d'amplitude supérieure à celles de quart-diurnes (M4, MS4) pouvant expliquer l'asymétrie. Un autre phénomène à prendre en considération est la composante résiduelle de courant, générée aussi par l'interaction non-linéaire des ondes principales de marée (PRANDLE, 2009). En termes de perspective, nous envisageons 
d'appliquer d'autres techniques d'analyse de données et d'intégrer l'information issue de la modélisation de la marée en mer d’Iroise afin d'étayer nos résultats basés sur l'utilisation des mesures de vitesse de courant par radar.

\section{Références bibliographiques}

ARDHUIN F., MARIE L., RASCLE N., FORGET P., ROLAND A. (2009). Observation and Estimation of Lagrangian, Stokes, and Eulerian Currents Induced by Wind and Waves at the Sea Surface. Journal of Physical Oceanography, Vol. 39(11). http://dx.doi.org/10.1175/2009JPO4169.1

EMERY W.J., THOMSON R.E. (Eds.). (2001). Data analysis methods in physical oceanography. Elsevier.

GONELLA J. (1972). A rotary-component method for analysing meteorological and oceanography vector time series. Deep Sea Res., Vol. 19, pp 833-846.

HOULSBY G.T., DRAPER S., OLDFIELD M.L.G. (2008). Application of linear momentum actuator disc theory to open channel flow. Report no. OUEL, 2296(08).

KOROTENKO K., SENTCHEV A., SCHMITT F.G., JOUANNEAU N. (2013). Variability of turbulent quantities in the tidal bottom boundary layer: Case study in the eastern English Channel. Continental Shelf Research, Vol. 58, pp 21-31. http://dx.doi.org/10.1016/j.csr.2013.03.001

LE BOYER A., CAMBON G., DANIAULT N., HERBETTE S., LE CANN B., MARIE L., MORIN P. (2009). Observations of the Ushant tidal front in September 2007. Continental Shelf Research, Vol. 29, pp 1026-1037.

LE PROVOST C. (1991). Generation of overtides and compound tides (pp. 269-296). John Wiley and Sons, New York.

MULLER H., F. DUMAS B. BLANKE MARIETTE V. (2009). Estimating the Lagrangian residual circulation in the Iroise Sea. J. Mar. Systems, 78, 1, S17-S36.

PADUAN J.D., WASHBURN L. (2013). High-frequency radar observations of ocean surface currents. Annual review of marine science, Vol. 5, pp 115-136. http://dx.doi.org/10.1146/annurev-marine-121211-172315

PRANDLE D. (1991). A new view of near-shore dynamics based on observation from HF radar. Progress in Oceanography, Vol. 27(3), pp 403-438. http://dx.doi.org/10.1016/00796611(91)90030-P

PRANDLE D. (2009). Estuaries: dynamics, mixing, sedimentation and morphology. Cambridge University Press. http://dx.doi.org/10.1017/CBO9780511576096

RAMOS V., CARBALLO R., ALVAREZ M., SANCHEZ M., IGLESIAS G. (2013). Assessment of the impact of tidal stream energy through high-resolution numerical modeling. Energy, Vol. 61, pp 541-554. http://dx.doi.org/10.1016/j.energy.2013.08.051

SENTCHEV A., FORGET P., BARBIN Y., YAREMCHUK M. (2013). Surface circulation in the Iroise Sea (W. Brittany) from high resolution HF radar mapping. Journal of Marine Systems, Vol. 109, S153-S168. http://dx.doi.org/10.1016/j.jmarsys.2011.11.024 